\title{
INTERFERENCE ALIGNMENT IN CLUSTERED AD HOC NETWORKS: HIGH RELIABILITY REGIME AND PER-CLUSTER ALOHA
}

\author{
Roland Tresch $\quad$ Giusi Alfano ${ }^{\star} \quad$ Maxime Guillaud ${ }^{\ddagger}$ \\ ${ }^{\dagger}$ FTW (Telecommunications Research Center Vienna), Vienna, Austria, email: tresch@ftw.at \\ ${ }^{\star}$ Dipartimento di Elettronica, Politecnico di Torino, Italy, email: alfano@tlc.polito.it \\ ${ }^{\ddagger}$ Institute of Telecommunications, Vienna University of Technology, Vienna, Austria, email: guillaud@tuwien.ac.at
}

\begin{abstract}
Wireless networks are fundamentally limited by the intensity of the received signals and the mutual interference caused by many concurrent transmissions. We consider large wireless ad hoc networks whose underlying node distribution is a Poisson cluster process. Under the assumption that multiple antennas are available at each node, we evaluate the performance of interference alignment (IA) inside each cluster. We extend our previous analysis of the outage probability achieved by this technique by generalizing it to IA with receive diversity. Furthermore, we provide a simplified expression applicable at high Signal-to-Interference Ratio, along the lines of the analysis recently proposed by Ganti, Andrews and Haenggi.
\end{abstract}

Index Terms - Interference Alignment, Stochastic Geometry

\section{INTRODUCTION}

Interference determines the fundamental performance limits of wireless ad hoc networks. In that setting, the exact characterization of the interference including fading effects and general node distributions is a challenging problem. Analytically tractable results on the interference distribution, outage probability and transmission capacity of large random wireless networks whose underlying node distribution is a Poisson clustered process were established in [1]. In this work, techniques from stochastic geometry were used to characterize the distribution of interference at a reference receiving node not belonging to the spatial point process as a function of the density of the transmitters, the path-loss function and the fading distribution.

Interference alignment (IA) over the $K$-user interference channel was introduced in [2], where it was shown to achieve almost surely a sum-rate multiplexing gain of $\frac{K}{2}$ per time and frequency dimension. This coding technique, requiring perfect channel knowledge, is based on linear precoding at the transmitters and interference suppression filtering at the receivers. By aligning all interfering signals in the same subspace from the point of view of all receivers simultaneously, IA enables interference-free communication over a channel of reduced dimension.

Despite the fact that IA over the constant MIMO interference channel is only possible among a limited number of users [3], we proposed in [4] to use IA in infinite networks, by aligning interference only inside finite-sized clusters of nodes, and suffering from

This work was supported in parts by the Vienna Science and Technology Fund (WWTF) through the project COCOMINT of FTW, by the COMET competence center program of the Austrian government and the City of Vienna through the FTW I0 project, by the Austria Science Fund (FWF) through grant NFN SISE (S106), and by the FP7 Newcom++ and TREND networks of excellence of the European Commission. uncoordinated interference from the rest of the network. Other attempts at applying IA to large networks can be found e.g. in [5] and references therein. These results are not directly comparable to our work: [5] assumes a finite area, while we consider an infinite space, multiple-antenna terminals and different fading assumptions.

The goal of this contribution is to evaluate the performance of MIMO interference alignment in large clustered wireless networks, while accounting for fading. The contributions are as follows:

- We extend the previous outage probability results from [4] to the case of IA with receive diversity [3], i.e. where the dimension of the intra-cluster interference-free signal space available at the receiver can be strictly greater than the rank of the signal of interest.

- We link this analysis to the high Signal-to-Interference Ratio (SIR) framework of [6], whereby the probability of nonoutage when using ALOHA can be written as $\mathbb{P}$ (success) $1-\gamma \eta$ as the density of interferers $\eta$ goes to zero, and identify the spatial contention parameter $\gamma$ applicable to the percluster IA model.

- We compare the performance of alignment-based precoding with dominant eigenmode (EM) transmission, analyzed in [7] for a homogeneous node distribution.

\section{SYSTEM MODEL}

Let us introduce the model for the clustered wireless ad hoc network. The clustering of nodes may be due to geographical factors, hot spots with a high user density or induced by the channel access scheme. The location of the transmitting nodes is modeled as a Neyman-Scott cluster process which is a stationary and isotropic Poisson cluster process $\Phi$ on the infinite plane $\mathbb{R}^{2}$, an example of which is depicted in Fig. 11 A Neyman-Scott process results from homogeneous independent clustering applied to a stationary Poisson process [1]. The cluster process consists of a parent Poisson process of intensity $\lambda_{p}$. The parent points identify the cluster centers (depicted by crosses in Fig. 11 and serve as reference points for the daughter points (transmitters) that are scattered i.i.d around the parent point according to a circularly symmetric normal distribution of variance $2 \sigma^{2}$. Here, we assume that the number of points $K$ in a representative cluster $\Psi$ is fixed. The clusters form a partition of $\Phi$. The overall intensity of the cluster process is $\lambda=\lambda_{p} K$.

We can write the point process as a random set $\Phi=\left\{x_{1}, x_{2}, \ldots\right.$, $\left.x_{\infty}\right\}$, where $x_{j}$ is the coordinate of the point in $\mathbb{R}^{2}$. Let us denote the set of indices of the points $\phi=\{1,2, \ldots, \infty\}$ where $\psi=$ $\{k, k+1, \ldots, k+K-1\}$ is a subset of $\phi$ containing the indices of $\Psi$. Each transmitter $j \in \phi$ is assumed to transmit at unit power. 


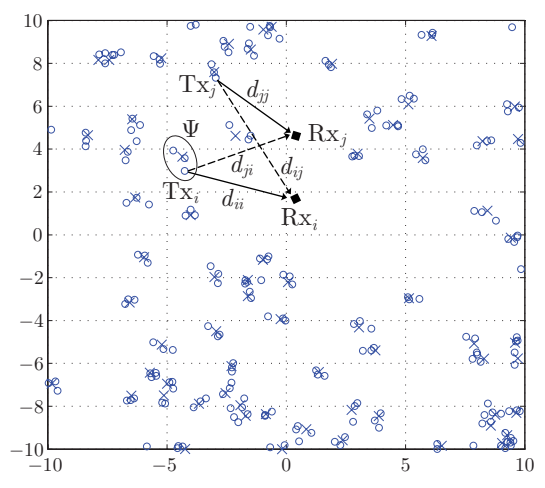

Fig. 1. Poisson cluster process with $\lambda_{p}=0.2, K=3, \sigma=0.25$

The receivers, depicted by squares in Fig. 1, are not considered a part of the process. That is, the model excludes the partner selection problem and focuses on the notion of a common distance that information travels in the network [1], aka the bipolar model. Every transmitter and receiver is equipped with $N_{\mathrm{T}}$ and $N_{\mathrm{R}}$ antennas, respectively. The transmitters inside of a cluster $\Psi$ use a spatial IA scheme, i.e. the members of a cluster $\Psi$ cooperate and adjust the spatial structure of their transmitted signals in order to avoid interfering with each other. The rest of the network $\Phi \backslash \Psi$ contributes non-aligned interference. While not optimal in general, treating the inter-cluster interference as noise is, in fact, optimal in the Gaussian weak interference regime. In particular, this case arises when the cluster intensity $\lambda_{p}$ is small.

Let us focus on a receiver $i \in \phi$, see Fig. 1 . The discrete-time signal received at a given time instant is the superposition of the signals transmitted by the $K$ transmitters of the considered cluster $\Psi$, and the transmitters of the rest of the network $\Phi \backslash \Psi$, weighted by their respective channel gains and path-loss coefficients. Namely, the signal at receiver $i$ can be written as

$$
\underline{\mathbf{y}}_{i}=\sum_{j \in \phi} \sqrt{\gamma_{i j}} \mathbf{H}_{i j} \underline{\mathbf{v}}_{j} s_{j}+\underline{\mathbf{n}}_{i},
$$

where $s_{j} \in \mathbb{C}$ represents the scalar signal transmitted by node $j$, and $\underline{\mathbf{v}}_{j} \in \mathbb{C}^{N_{\mathrm{T}} \times 1}$ is the associated precoding vector which will be further specified in Subsection 2.1 $\left[\mathbf{H}_{i j}\right]_{i, j \in \phi}$ are complex $N_{\mathrm{R}} \times N_{\mathrm{T}}$ matrices representing the MIMO channels between transmitter $j$ and receiver $i$. We assume a flat-fading channel model and Rayleigh fading, i.e. the elements of $\mathbf{H}_{i j}$ are complex Gaussian i.i.d. random variables, with zero mean and unit variance. $\gamma_{i j}=g\left(d_{i j}\right)$ is the path-loss model on the same link with $d_{i j}$ the distance between transmitter $j$ and receiver $i$ and $g(\cdot)$ the path-loss function. $\underline{\mathbf{n}}_{i}$ is additive white Gaussian noise.

\subsection{Intra-cluster IA and Feasible Cluster Settings}

Let us focus on a given cluster $\Psi$, where we aim to achieve IA with multiplexing gain allocation 1 for each of the $K$ users in $\Psi$. This strategy is suboptimal in general, but chosen for analytical tractability. Each transmitter is assigned a precoding vector $\underline{\mathbf{v}}_{i}$ in order to steer its transmitted signal into a receive signal subspace of minimum dimension at each unintended intra-cluster receiver. IA is feasible in $\Psi$ with multiplexing gain 1 and receive diversity $d^{\prime}$ per user iff there exists $N_{\mathrm{T}} \times 1$ unit-norm vectors (precoding vectors) $\underline{\mathbf{v}}_{i}$ and $N_{\mathrm{R}} \times d^{\prime}$ truncated unitary projection matrices (interference suppres- sion matrices) $\mathbf{U}_{i}$ such that, for all $i \in \psi$,

$$
\begin{aligned}
\mathbf{U}_{i}^{\mathrm{H}} \mathbf{H}_{i j} \underline{\mathbf{v}}_{j} & =\mathbf{O}_{d^{\prime} \times 1}, \forall(j \neq i) \in \psi, \quad \text { and } \\
\operatorname{rank}\left(\mathbf{U}_{i}^{\mathrm{H}} \mathbf{H}_{i i} \underline{\mathbf{v}}_{i}\right) & =1 .
\end{aligned}
$$

As shown in [3. Theorem 1], under mild assumptions on the channel fading process, and considering a network where all nodes are equipped with respectively $N_{\mathrm{T}}$ transmit or $N_{\mathrm{R}}$ receive antennas, a solution (in terms of the $\mathbf{U}_{i}$ and $\underline{\mathbf{v}}_{i}$ for $i \in \psi$ ) to the intra-cluster IA problem above exists almost surely iff

$$
\frac{N_{\mathrm{T}}-1}{d^{\prime}}+N_{\mathrm{R}}-d^{\prime}+1 \geq K \text {. }
$$

Henceforth, we assume that 4 is satisfied. Note that each transmitter causes uncoordinated interference to all receivers outside its cluster, i.e. $i \in(\phi \backslash \psi)$.

Let us now introduce a receiver architecture that uses linear operations to suppress the intra-cluster interference and to achieve the feasible diversity gain. The intra-cluster interference decorrelator is a linear filter comprised of a projection receiver followed by the matched filter (MF) to the signal of interest in the intra-cluster interference-free subspace, i.e. $\underline{\mathbf{u}}_{\mathrm{D} i}=\mathbf{U}_{i} \underline{\mathbf{u}}_{\mathrm{MF} i}$, with $\mathbf{U}_{i} \in \mathbb{C}^{N_{\mathrm{R}} \times d^{\prime}}$ as defined in 22 and $\underline{\mathbf{u}}_{\mathrm{MF} i}=\mathbf{U}_{i}^{\mathrm{H}} \mathbf{H}_{i i} \underline{\mathbf{v}}_{i} /\left\|\mathbf{U}_{i}^{\mathrm{H}} \mathbf{H}_{i i} \underline{\mathbf{v}}_{i}\right\| \in \mathbb{C}^{d^{\prime} \times 1}$.

The following example with $d^{\prime}=2$ and $N_{\mathrm{R}}=3$ illustrates the interference decorrelator. The three-dimensional receive signal space of user $i$ in the cluster $\Psi$ is shown in Fig. 2 The intracluster interference $\left[\sqrt{\gamma_{i k}} \mathbf{H}_{i k} \underline{\mathbf{v}}_{k} s_{k}\right]_{k \neq i \in \psi}$ is aligned along a onedimensional subspace. The intra-cluster interference decorrelator projects the receive signal onto the plane orthogonal to the span of $\left[\sqrt{\gamma_{i k}} \mathbf{H}_{i k} \underline{\mathbf{v}}_{k} s_{k}\right]_{k \neq i \in \psi}$. The projection is depicted along the dashed lines and the components of the signal of interest $\sqrt{\gamma_{i i}} \mathbf{H}_{i i} \mathbf{v}_{i} s_{i}$ and the inter-cluster interference $\left[\sqrt{\gamma_{i k}} \mathbf{H}_{i j} \underline{\mathbf{v}}_{j} s_{j}\right]_{j \in(\phi \backslash \psi)}$ parallel to the plane are highlighted in Fig. 2 The projection of the signal of interest $\sqrt{\gamma_{i i}} \mathbf{U}_{i}^{\mathrm{H}} \mathbf{H}_{i i} \underline{\mathbf{V}}_{i} s_{i}$ spans a one dimensional subspace in the two dimensional intra-cluster interference-free subspace of user $i$. The MF to the signal of interest achieves the diversity gain and further mitigates the energy of the residual inter-cluster interference.

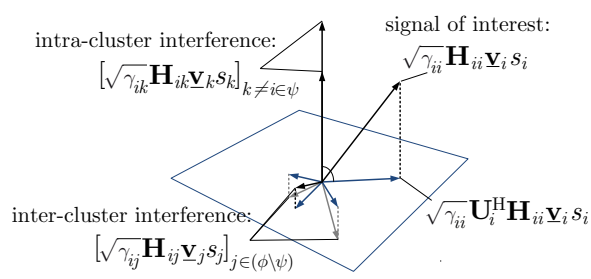

Fig. 2. Intra-cluster interference decorrelator

Using the interference decorrelator, the filtered receive signal $\bar{y}_{i}=\underline{\mathbf{u}}_{\mathrm{D} i}^{\mathrm{H}} \underline{\mathbf{y}}_{i}$ writes

$$
\begin{aligned}
\bar{y}_{i} & =\sqrt{\gamma_{i i}}\left\|\mathbf{U}_{i}^{\mathrm{H}} \mathbf{H}_{i i} \underline{\mathbf{v}}_{i}\right\| s_{i}+\sum_{j \in(\phi \backslash \psi)} \sqrt{\gamma_{i j}} \underline{\mathbf{u}}_{\mathrm{D} i}^{\mathrm{H}} \mathbf{H}_{i j} \underline{\mathbf{v}}_{j} s_{j}+\underline{\mathbf{u}}_{\mathrm{D} i}^{\mathrm{H}} \underline{\mathbf{n}}_{i} \\
& =\sqrt{\gamma_{i i}} \bar{h}_{i i} s_{i}+\sum_{j \in(\phi \backslash \psi)} \sqrt{\gamma_{i j}} \bar{h}_{i j} s_{j}+\bar{n}_{i}
\end{aligned}
$$

where interference from transmitters within $\Psi$ is perfectly suppressed, due to 22. The effective channel $\bar{h}_{i i}=\left\|\mathbf{U}_{i}^{\mathrm{H}} \mathbf{H}_{i i} \underline{\mathbf{v}}_{i}\right\|$ as seen by receiver $i$ is scalar. Furthermore, since $\mathbf{U}_{i}^{\mathrm{H}} \mathbf{U}_{i}=\mathbf{I}_{d^{\prime}}$ and $\underline{\mathbf{v}}_{i}^{\mathrm{H}} \underline{\mathbf{v}}_{i}=1$, 
and $\mathbf{U}_{i}, \underline{\mathbf{v}}_{i}$ are independent of $\mathbf{H}_{i i}, \mathbf{U}_{i}^{\mathrm{H}} \mathbf{H}_{i i} \underline{\mathbf{v}}_{i}$ has $d^{\prime}$ Gaussian i.i.d. fading coefficients having the same variance as the components in $\mathbf{H}_{i i}$. Hence, $\left\|\mathbf{U}_{i}^{\mathrm{H}} \mathbf{H}_{i i} \mathbf{v}_{i}\right\|$ is Chi distributed with $2 d^{\prime}$ degrees of freedom. Similarly, since $\underline{\mathbf{u}}_{\mathrm{D} i}^{\mathrm{H}} \underline{\mathbf{u}}_{\mathrm{D} i}=\underline{\mathbf{u}}_{\mathrm{MF} i}^{\mathrm{H}} \mathbf{U}_{i}^{\mathrm{H}} \mathbf{U}_{i} \underline{\underline{\mathrm{u}}}_{\mathrm{MF} i}=1$ and independent of $\left[\mathbf{H}_{i j}\right]_{j \in \phi \backslash \psi}$, the effective channels $\overline{\bar{h}}_{i j}=\underline{\mathbf{u}}_{\mathrm{D} i}^{\mathrm{H}} \mathbf{H}_{i j} \underline{\mathbf{v}}_{j}$ are scalar Gaussian fading with the same variance as the components of $\mathbf{H}_{i j}$. The effective noise term $\bar{n}_{i}=\underline{\mathbf{u}}_{\mathrm{D} i}^{\mathrm{H}} \underline{\mathbf{n}}_{i}$, is a scalar Gaussian fading with the same variance as the noise vector $\underline{\mathbf{n}}_{i}$.

\section{OUTAGE PROBABILITY AND HIGH RELIABILITY ANALYSIS}

In this section, we introduce the model for successful transmission, review the expression for intra-cluster IA without diversity gain derived in [4] and analyze the performance of intra-cluster IA with receive diversity in the high SIR regime.

We assume that the desired transmitter is located at the origin and its intended receiver at location $z$ at distance $d_{i i}=\|z\|$. Communication is assumed successful iff the SIR exceeds a threshold $T$ that depends on the physical layer parameters such as rate of transmission, modulation and coding. The probability of success for this pair (neglecting the noise) is given by [6]

$$
\mathbb{P}(\text { success })=\mathbb{P}\left(\frac{\left|\bar{h}_{i i}\right|^{2} \gamma_{i i}}{I_{\phi \backslash \psi}} \geq T\right),
$$

with $I_{\phi \backslash \psi}=\sum_{j \in(\phi \backslash \psi)}\left|\bar{h}_{i j}\right|^{2} \gamma_{i j}$ the accumulated inter-cluster interference from the rest of the network with $\left|\bar{h}_{i j}\right|^{2}$ exponentially distributed. The complementary cumulative density function (CCDF) of the signal power of interest is $F_{\left|\bar{h}_{i i}\right|^{2}}(h)=e^{-h} \sum_{k=0}^{d^{\prime}-1} h^{k} / k$ !. Using tools from stochastic geometry, a numerically integrable expression for the case of $d^{\prime}=1$ is derived in [4, Equation (9)]. A closed form upper bound on the probability of success is derived for the path-loss model $g\left(d_{i j}\right)=d_{i j}^{-\alpha}$ with $\alpha=4$ and small cluster sizes

$$
\mathbb{P}(\text { success }) \leq \exp \left\{-\lambda_{p} \pi \delta(K) d_{i i}^{2} \sqrt{T} \tan ^{-1}\left(\frac{d_{i i}^{2} \sqrt{T}}{4 \sigma^{2}}\right)\right\},
$$

where $\delta(K)=\sum_{k=0}^{K-1}(-1)^{k}\left(\begin{array}{c}-1 / 2 \\ k\end{array}\right)$. The generalization of (7) to intra-cluster IA with receive diversity $d^{\prime}$ is not straightforward. However, the outage probability can be analyzed in the highreliability regime for any $d^{\prime}$. As the underlying MAC protocol, we assume highly clustered ALOHA [8], i.e. each cluster either transmits or remains muted with a fixed medium access probability $\eta$. This is equivalent to a thinning of the parent Poisson point process.

Let us denote the parent process $\Phi_{p}=\left\{x_{p 1}, x_{p 2}, \ldots x_{p \infty}\right\}$ with $x_{p i}$ the coordinate of the cluster center and $\phi_{p}=\{1,2, \ldots, \infty\}$ is the set of indices of the clusters. In the asymptotic regime as the density of residual inter-cluster interferers goes to zero, we use a cluster fusion approach to derive the intrinsic spatial contention of the network. Hence, we replace the daughter points by a representative point at the coordinates of the cluster center. Assuming finite cluster size, the accumulated interference in 6 is approximated by

$$
I_{\phi \backslash \psi} \approx \sum_{j \in \phi_{p} \backslash i}\left|\bar{h}_{i j}^{\prime}\right|^{2} \gamma_{i j}^{\prime}
$$

with $\left|\bar{h}_{i j}^{\prime}\right|^{2}=\sum_{k \in \Psi_{j}}\left|\bar{h}_{i k}\right|^{2}$ the compound channel power gain and $\gamma_{i j}^{\prime}=g\left(\left\|z-x_{p j}\right\|\right)$ the path-loss coefficient. $\left|\bar{h}_{i j}^{\prime}\right|^{2}$ is the sum of i.i.d. exponentially distributed random variables and therefore Gamma distributed, i.e. the density function writes $f_{\left|\bar{h}_{i j}^{\prime}\right|^{2}}(h)=$ $h^{K-1} \exp (-h) / \Gamma(K) . \Phi_{p}$ is a homogeneous Poisson point process with second order product density $\rho^{(2)}(x)=\lambda_{p}^{2}$ and according to 6 , Theorem 3] the asymptotic success probability writes $\mathbb{P}($ success $) \sim$ $1-\gamma \eta$ with the spatial contention

$$
\gamma=\frac{1}{\lambda_{p}} \int_{\mathbb{R}^{2}}\left[1-\mathbb{E}_{\left|\bar{h}_{i j}^{\prime}\right|^{2}} F_{\left|\bar{h}_{i i}\right|^{2}}\left(h \frac{T g(x)}{g\left(d_{i i}\right)}\right)\right] \rho^{(2)}(x) \mathrm{d} x .
$$

The integrand in 9) can be simplified to

$$
\begin{aligned}
& \lambda_{p}\left[1-\int_{0}^{\infty} F_{\left|\bar{h}_{i i}\right|^{2}}\left(h^{\prime}\right)(h \vartheta)\left(\frac{h^{\prime}}{\vartheta}\right)^{K-1} e^{-h^{\prime} / \vartheta} \frac{1}{\Gamma(K) \vartheta} \mathrm{d} h^{\prime}\right] \\
= & \lambda_{p}\left[1-\frac{1}{\Gamma(K) \vartheta^{K}}\left(\left.\frac{\mathrm{d}}{\mathrm{d} K-1}(-1)^{K-1} \mathcal{L}\left(F_{\left|\bar{h}_{i i}\right|^{2}}(s)\right)\right|_{s=\frac{1}{\vartheta}}\right)\right]
\end{aligned}
$$

$=\lambda_{p}\left[1-\frac{1}{\Gamma(K) \vartheta^{K}}\left(\left.\frac{\mathrm{d}}{\mathrm{d} K-1}(-1)^{K-1} \sum_{k=0}^{d^{\prime}-1} \frac{1}{(1+s)^{k+1}}\right|_{s=\frac{1}{\vartheta}}\right)\right]$,

with $\vartheta=T g(x) / g\left(d_{i i}\right)$. For the first expression we used the substitution $h^{\prime}=h \vartheta$, the first equality comes from the Laplace transform equivalence $t^{n} f(t) \longleftrightarrow(-1)^{n} \mathcal{L}(f)^{(n)}(s)$ and the second equality from the equivalence $\left(t^{n} / n !\right) e^{-t} \longleftrightarrow 1 /(s+1)^{n+1}$. Above steps turn 9 into

$$
\gamma=\lambda_{p} \int_{\mathbb{R}^{2}}\left[1-\left(\frac{g\left(d_{i i}\right)}{T g(x)}\right)^{K}\left(\sum_{k=0}^{d^{\prime}-1} \frac{\left(\begin{array}{c}
K-1+k \\
K-1
\end{array}\right)}{\left(1+\frac{g\left(d_{i i}\right)}{T g(x)}\right)^{K+k}}\right)\right] \mathrm{d} x .
$$

For the special case of $g\left(d_{i j}\right)=d_{i j}^{-\alpha}$ with $\alpha=4$, one can show (steps omitted) that 11 is equivalent to

$$
\gamma=\lambda_{p} \pi^{2} / 2 \delta(K) \epsilon\left(d^{\prime}\right) d_{i i}^{2} \sqrt{T},
$$

with $\delta(K)$ from 77 and $\epsilon\left(d^{\prime}\right)=\left(\begin{array}{c}2\left(d^{\prime}-1\right) \\ d^{\prime}-1\end{array}\right) / 4^{d^{\prime}-1}$. Hence, $\epsilon\left(d^{\prime}\right)$ in 12 captures the impact of diversity gain on the success probability. We note that for $d^{\prime}=1,1-\gamma \eta$ with $\gamma$ according to 12 coincides with a first-order Taylor approximation of (7) for $\sigma \rightarrow 0$.

\section{SIMULATION RESULTS}

In this section, we present simulation results and validate the high SIR formulas derived above. The curves for the probability of success are verified by Monte-Carlo simulation of (6) over different transmitter positions and fading coefficients. The standard power law $g\left(d_{i j}\right)=d_{i j}^{-\alpha}$ with $\alpha=4$ and a threshold $T=1$ is used.

We evaluate the transmission success probability of intra-cluster IA with receive diversity and compare with the outage performance of dominant EM transmission. For dominant EM transmission, $\left|\bar{h}_{i i}\right|^{2}$ in 6 is distributed as the largest eigenvalue of a complex Wishart matrix whereas the interference term contains inter- and intra-cluster interference, i.e. $I_{\phi}=\sum_{j \neq i \in \phi}\left|\bar{h}_{i j}\right|^{2} \gamma_{i j}$ with $\left|\bar{h}_{i j}\right|^{2}$ exponentially distributed [7].

The results are presented in Fig. 3 for a network with fixed cluster density $\lambda_{p}=0.05$ and cluster scattering parameter $\sigma=0.25$. 
Depending on $d^{\prime}$ and the antenna configuration, a different number of users per cluster can be accommodated according to (4). Here, we consider the case of $N_{\mathrm{R}}=3$ receive antennas and two different transmit antenna deployments, i.e. $N_{\mathrm{T}}=5$ and 7 . We consider the case of $K=4$ users allowing for $d^{\prime}=2$ and $K=7$ users with $d^{\prime}=1$ for the $5 \times 3$ antenna setting, as well as $K=5$ users rendering $d^{\prime}=2$ and $K=9$ users with $d^{\prime}=1$ for the $7 \times 3$ antenna setting, respectively. All cases fulfill the feasibility criterion (4) with equality. In all cases considered, intra-cluster IA outperforms dominant

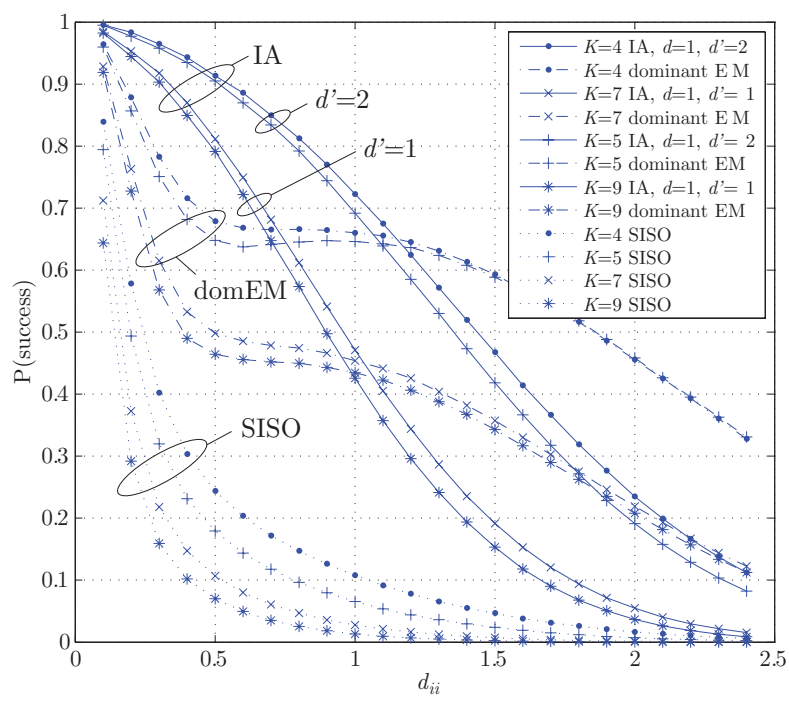

Fig. 3. $\mathbb{P}$ (success) versus $d_{i i}$ for $\lambda_{p}=0.05, \sigma=0.25$

EM transmission for small transmitter-receiver distances $d_{i i}$. IA with receive diversity substantially improves the transmission success probability. For a fixed outage probability target, the maximum achievable $d_{i i}$ is increased significantly for both antenna configurations, e.g. $\mathbb{P}$ (success) $>0.9$, the maximum $d_{i i}$ increases by about $60 \%$. However, decreasing diversity allows for more users per cluster that can be supported while still fulfilling the IA condition. Dominant EM outperforms intra-cluster IA for large distances between transmitter-receiver pairs. Here, the inter-cluster interference is predominant and the performance of intra-cluster IA is suffering from the diminishing received intended signal power caused by the alignment procedure. In that case, maximizing the receive signal power of the intended transmitter should be preferred to IA. In all considered cases, the equivalent SISO system performs worst. Clearly, an increasing number of nodes per cluster decreases the transmission success probability, as can be verified from Fig. 3

For the same network settings as above, Fig. 4 plots the derived asymptotic expression $1-\gamma \eta$ for intra-cluster IA with diversity gain $d^{\prime}=1,2$ with $\gamma$ according to 12 and $d_{i i}=2$ along with MonteCarlo simulations. We observe a close match between simulation and asymptotic expression for small $\eta$.

\section{CONCLUSION}

The performance of intra-cluster interference alignment with receive diversity was characterized in a large clustered wireless ad hoc network. The proposed scheme was shown to significantly increase the reliability of short range communication with respect to non-

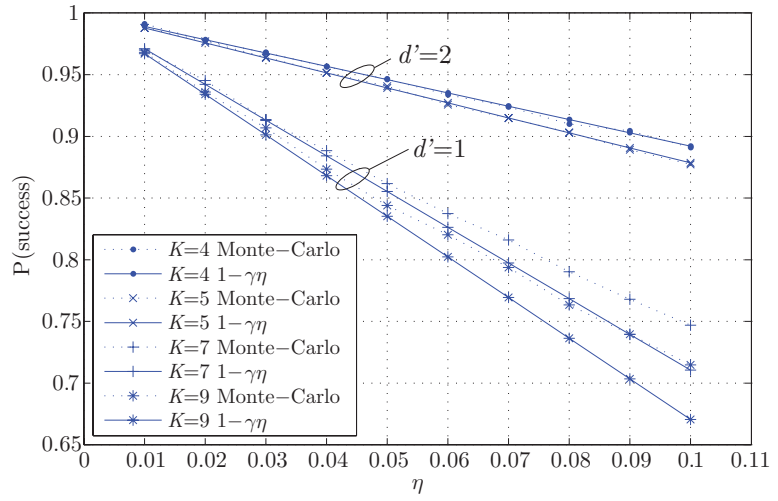

Fig. 4. $\mathbb{P}$ (success) versus $\eta$

cooperative precoding schemes. Interference alignment in conjunction with per cluster ALOHA was analyzed in the high-reliability regime. For a special network setting, closed form expressions for the outage probability were derived and the term related to the diversity gain was identified.

\section{REFERENCES}

[1] Radha Krishna Ganti and Martin Haenggi, "Interference and outage in clustered wireless ad hoc networks," IEEE Trans. Information Theory, vol. 55, no. 9, pp. 4067-4086, Sept. 2009.

[2] Viveck R. Cadambe and Syed A. Jafar, "Interference alignment and degrees of freedom of the $K$-user interference channel," IEEE Trans. Information Theory, vol. 54, no. 8, pp. 34253441, Aug. 2008.

[3] M. Guillaud, "Receive diversity and ergodic performance of interference alignment on the MIMO Gaussian interference channel," in Proc. Allerton Conference on Communication, Control, and Computing, Monticello, IL, USA, Sept. 2010.

[4] Roland Tresch and Maxime Guillaud, "Performance of interference alignment in clustered wireless ad hoc networks," in Proc. IEEE Int. Symp. on Information Theory (ISIT), Austin, Texas, USA, June 2010.

[5] Oliver Johnson, Matthew Aldridge, and Robert Piechocki, "Interference alignment-based sum capacity bounds for random dense Gaussian interference networks," IEEE Trans. Information Theory, vol. 57, no. 1, pp. 282 -290, Jan. 2011.

[6] R. K. Ganti, J. G. Andrews, and M. Haenggi, "High-SIR transmission capacity of wireless networks with general fading and node distribution," IEEE Trans. Information Theory, 2010, Submitted.

[7] A.M. Hunter, J. Andrews, and S. Weber, "Transmission capacity of ad hoc networks with spatial diversity," Wireless Communications, IEEE Transactions on, vol. 7, no. 12, pp. 5058 -5071, dec. 2008.

[8] Riccardo Giacomelli, Rhadha Krishna Ganti, and Martin Haenggi, "Outage probability of general ad hoc networks in the high-reliability regime," IEEE/ACM Transactions on Networking, 2010, Submitted. 